\title{
An unusual intraosseous calcaneal lipoma: case report
}

\author{
Ender Alkan', Hasan Erdoğan², Turgay Kara² \\ ${ }^{1}$ Department of Radiology, Aksaray University School of Medicine, Aksaray, Turkey \\ ${ }^{2}$ Department of Radiology, Konya Training and Research Hospital, Konya, Turkey
}

DOI: $10.18621 /$ eurj.360318

\begin{abstract}
Intraosseous lipoma, a rare primary benign tumor of the skeleton, is most commonly found in the calcaneus. It may contain homogenous fat, but it may also contain necrosis, calcification, or ossification. It usually does not show contrast enhancement, but there is an interface enhancement between the outer fat plan and the inner fluid part. Herein, we present an unusual 22-year-old male case of calcaneal intraosseous lipoma with contrast enhancement in the fatty component. The lesion was totally curetted and replaced with bone graft. The pathological diagnosis was reported as an intraosseous lipoma. The correct diagnosis of an atypical calcaneal intraosseous lipoma is very important to prevent an unnecessary biopsy and treatment.
\end{abstract}

Keywords: Intraosseous lipoma, calcaneus, magnetic resonance imaging

Received: December 2, 2017; Accepted: March 8, 2018; Published Online: April 3, 2018

\begin{abstract}
T ipoma of bone is a benign neoplasm of Jadipocytes that typically arises within the medullary cavity of bone. Although there is a large amount of fatty tissue in bone marrow, intraosseous lipoma occurs very rarely, with a $0.1-2.1 \%$ prevalence [1-3]. However, this probably is not the actual incidence number because the lesions are frequently asymptomatic. Calcaneus bone is reported to be the most frequent site of intraosseous lipoma [4]. Herein, we present an unusual case of calcaneal intraosseous lipoma with contrast enhancement in the fatty component.
\end{abstract}

\section{CASE PRESENTATION}

A 22-year-old male patient presented with pain on his right heel and no abnormality in his laboratory results. Direct X-ray revealed a lytic lesion surrounded by sclerosis in the medullary of the right calcaneus bone (Figure 1). A contrasted magnetic resonance image (MRI) of the right heel and ankle was performed for initial diagnosis, which was a simple bone cyst and intraosseous lipoma. A $33 \times 28 \times 24 \mathrm{~mm}$ mass, which had a hypo intense lobulated sclerotic rim on the T1 and T2 weighted sequences was discovered via MRI. There was a peripherally fatty component, which was hyper intense on T1 and T2 sequences and suppressed on fatty suppressed sequences and there was cystic-degenerated areas centrally. After the injection of contrast medium, contrast uptake in the peripheral fatty component was detected (Figure 2). Overall, the lesion was diagnosed as an intraosseous lipoma with these MRI findings. According to Milgram's classification, the lesion was graded as a stage 3 calcaneal lipoma because of necrosis, centrally cystic transformed areas and sclerosis in the wall. The patient underwent operation, and the lesion was totally curetted away and replaced with bone graft. The pathological diagnosis was reported as an intraosseous lipoma (Figure 3).

Address for correspondence: Ender Alkan, MD., Assistant Professor, Aksaray University School of Medicine, Department of Radiology, Nevsehir Cad. Zafer Sk. No: 125, Aksaray, Turkey

E-mail:drealkan@gmail.com,Phone:+9003822882900 


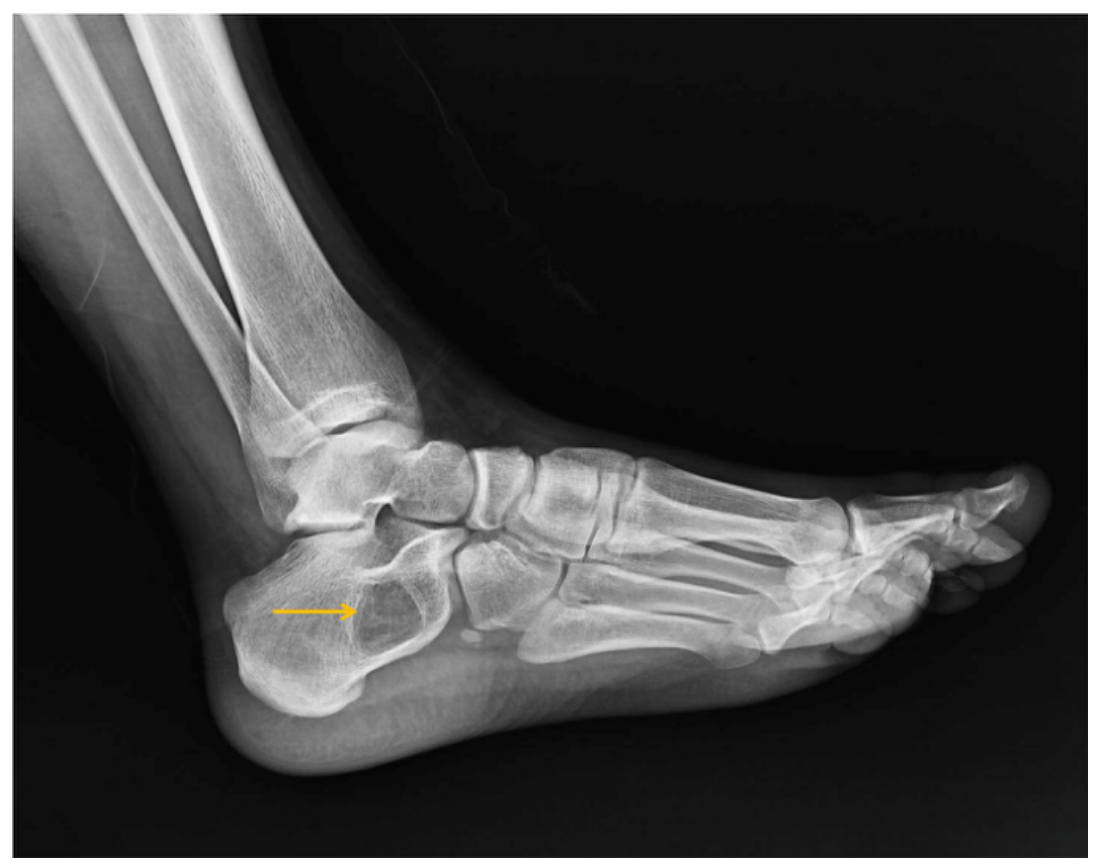

Figure 1. Right foot x-ray showing a centrally lytic, peripherally sclerotic lesion in the medulla of the calcaneus.
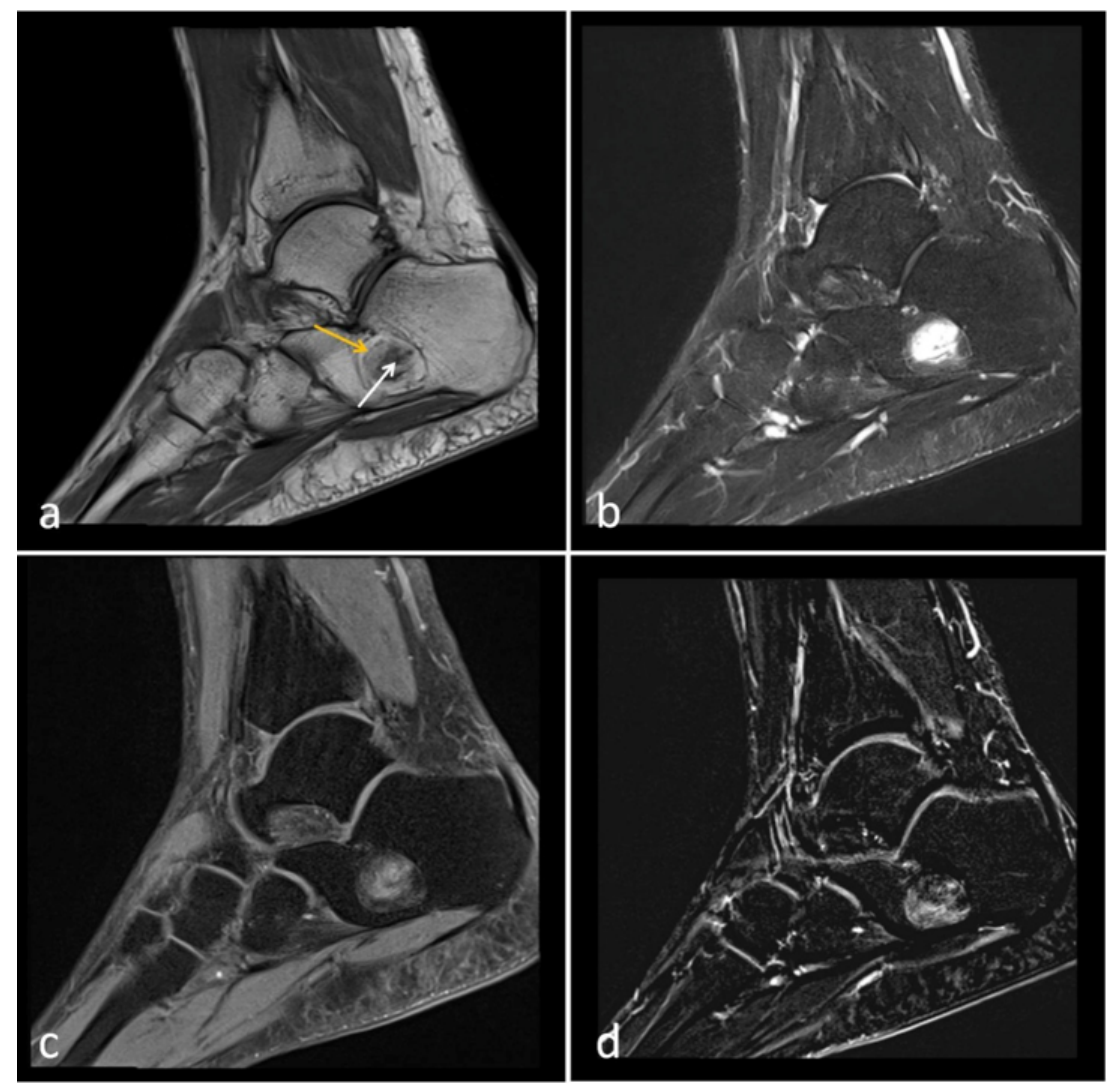

Figure 2. MRI study of the right ankle; there is hypo intense rim around the lesion in T1-weighted (a) and fat-suppressed T2 (b) weighted images consistent with sclerosis. Hyper intense fatty tissue is observed in the periphery of the lesion (yellow arrow) which is suppressed at fat-suppressed sequences $(b, c)$. Also there is a cystic component in the central of the lesion which is hypo intense in T1-weighted image (a) and hyper intense in T2-weighted image (b). In subtraction images (d), contrast enhancement is seen in the fat components after contrast medium injection. 


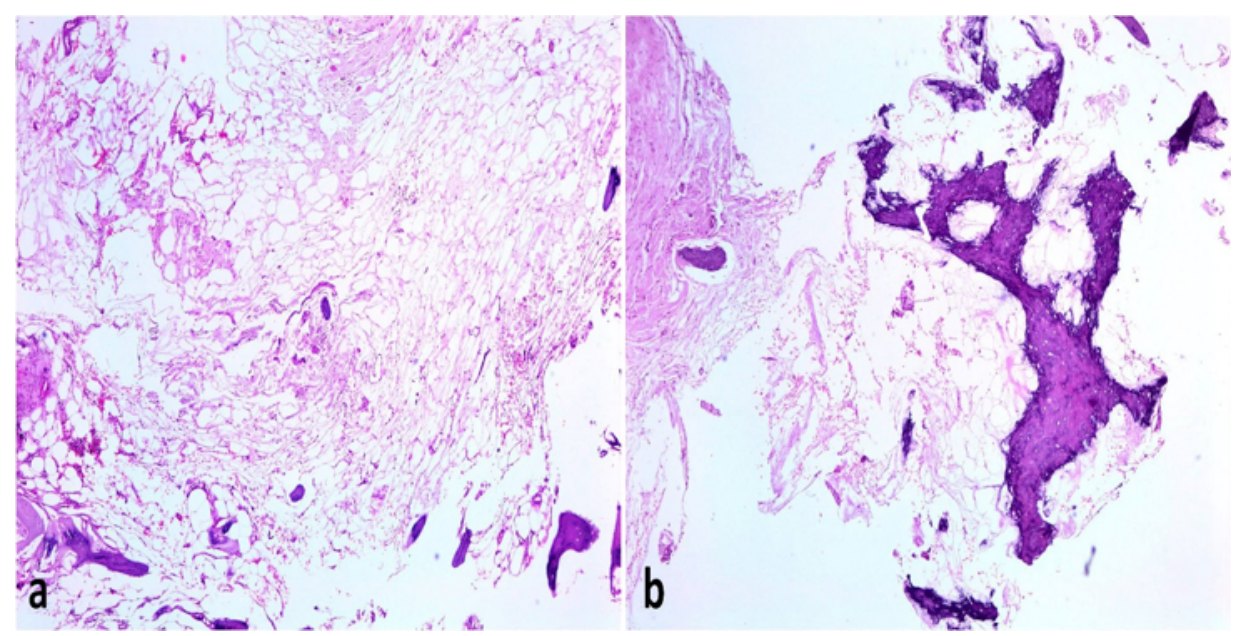

Figure 3. Intraosseous lipomas, microscopic image; (a) thin bone trabeculae are observed in the lower part. At the upper part, fat texture without cellular elements compatible with lipoma is observed. Hematoxylin and eosin (H\&E) stain, $\times 40$ magnification. (b) Dystrophic calcifications are seen on the intraosseous lipoma. H\&E stain, $\times 40$ magnification.

\section{DISCUSSION}

Intraosseous lipomas, which occur in both males and females, are generally present in the fourth and fifth decades of life, with a prevalence of $0.1-2.1 \%$. However, the real prevalence is thought to be higher than this estimate because most cases are asymptomatic [2, 3]. Therefore, most cases have been detected incidentally. Pain is the major complication in symptomatic patients, and they present with pathological fractures.

Although intraosseous lipoma can occur anywhere in the skeletal system, $71 \%$ of these lipomas are located in the lower extremities, and usually appears in the calcaneus $(32 \%)$, in which the most frequently found tumor is also the intraosseous lipoma. The upper extremities, skull, mandible, spine, and costs are involved with decreasing frequencies. Intraosseous lipomas located in long bones usually affect the metaphysis [5-8]. Basically, simple bone cysts called intraosseous ganglia are present in intraosseous lipomas. On X-rays, intraosseous lipomas appear as benign, osteolytic lesions with well-defined limits, and it is difficult to identify them only based on X-ray findings. Computerized tomography (CT), especially MRI, is useful in differential diagnosis. Radiographic diagnosis of a lipoma may not be straightforward and so there may be differential diagnosis that may include simple bone cyst, no ossifying fibroma, aneurysmal bone cyst, fibrous dysplasia, bone infarct, giant cell tumor, chondroid tumor or fungal infections. The appearance of these lesions on radiographs, CT scans and MRIs can vary as result of their degree of involution and necrosis. It is important to make a correct diagnosis on the basis of multi-method imaging studies, especially MRI.

In the classifications performed by Milgram et al. [4], intraosseous lipomas are divided into three stages based on fat necrosis: Stage 1A - sharply limited lesion with homogenous fat content; Stage 2 - Dominantly fatty lesions with central necrosis, calcification, or ossification, and Stage 3 - A heterogeneous, fatcontaining lesion involving multiple necrotic areas, cystic transformations, sclerosis, or ossification in the wall. Our case was staged as 3 according to this classification.

In intraosseous lipomas, there is usually no enhancement of contrast, and occasionally there is an interfacial enhancement between the external fat plan and the internal fluid. Contrast enhancement has not been reported in the inner and extra-lesional part of bones. Our case is important in that it demonstrates contrast enhancement in the inner part of the lesion, which has not been reported until now. Because calcaneal lipomas do not affect bone stability, conservative treatment can be applied. The fracture can be treated with curettage and bone graft in cases of doubt. There is usually no recurrence after surgery [5]. 


\section{CONCLUSION}

As a result, intraosseous lipomas can be diagnosed easily with their fatty component, especially via MRI. It is generally accepted that intraosseous lipomas are not contrasting, or that there is contrast enhancement only at the fat-liquid interface. However, as we have shown in our case, contrast enhancement can also be seen in the inner part of the lesion. The correct diagnosis of an atypical calcaneal intraosseous lipoma is very important to prevent an unnecessary biopsy and treatment.

\section{Informed consent}

Written informed consent was obtained from the patient for the publication of this case report.

\section{Conflict of interest}

The authors declared that there are no potential conflicts of interest with respect to the research, authorship, and/or publication of this article.

\section{REFERENCES}

[1] Campanacci M, Enneking WF. Bone and soft tissue tumors: clinical features, imaging, pathology and treatment. 2nd ed. Berlin: Springer, 1999, pp. 683-9.

[2] Myhre-Jensen OA. Consecutive 7-year series of 1331 benign soft tissue tumors. Clinicopathologic data. Comparison with sarcomas. Acta Orthop Scand 1981;52:287-93.

[3] Unni KK, Inwards CY. Dahlin's bone tumors: general aspect and data on 10165 cases. 6th ed. Philadelphia: Lippincott Williams \& Wilkins, 2006, pp. 298-300.

[4] Milgram JW. Intraosseous lipomas. Clin Orthop Relat Res 1988;231:277-302.

[5] Campbell RS, Grainger AJ, Mangham DC, Beggs I, Teh J, Davies AM. Intraosseous lipoma: report of 35 new cases and a review of the literature. Skeletal Radiol 2003;32:209-22.

[6] Blacksin MF, Ende N, Benvenia J. Magnetic resonance imaging of intraoeeous lipomas: a radiologic-pathologic correlation. Skletel Radiol. $1995 ; 24: 37-41$

[7] Bagatur AE, Yalcinkaya M, Dogan A, Gur S, Mumcuoglu E, Albayrak M. Surgery is not always necessary in intraosseous lipoma. Orthopedics 2010;33:306

[8] Hassani M, Gharehdaghi M, Khooei AR, Ghodsi E, Nazarzadeh H. Bilateral intraosseous tumor of the calcaneus with imaging-pathologic discordance: a case report and literatures review. Arch Bone Jt Surg 2014;2:238-42 